satz von 20 . - H ol t f r e te $\mathrm{r}^{4}$ fand bei ähnlichen Versuchen mit etwas caudaler gelegenen Abschnitten des Urdarmdaches in 9 von 30 Fällen neurale Differenzierungen aus Implantatmaterial $(=30 \%)$. Er führte dieses Ergebnis damals auf einen Versuchsfehler (Mitisolation von Medullarplatte) zurück, doch ist dies bei einiger Übung im Operieren von Neurulae praktisch ausgeschlossen. Immerhin ist dieser Prozentsatz grade für caudale Abschnitte ziemlich hoch. Dafür kann jedoch außer Versuchsfehlern auch das etwas geringere Alter der von $\mathrm{H}$ ol t f r e ter verwendeten Neurulae verantwortlich sein.

Unsere Beobachtung stellt einen neuen Beitrag zu dem zuerst von T. Y amada ${ }^{5}$ systematisch unter-

4 Roux Arch. 134, 523 [1936].

5 Roux Arch. 137, 151 [1938]. suchten Problem der Determination des Neurulamesoderms dar. Yamada stellte fest, daß das Rumpfmesoderm auch nach der Beendigung der Gastrulation noch ein ziemlich labiles System darstellt, indem sich die einzelnen mesodermalen Organanlagen (präsumptive Ursegmente, Vornieren, ventrales Mesoderm) gegenseitig vertreten können, sich also noch nicht potentiell voneinander unterscheiden. Die feste Determination soll erst mit dem Erscheinen der Medullarwülste einsetzen. Unsere Beobachtung zeigt, daß das meso dermale Material darüber hinaus - selbst noch im Medullarplattenstadium - Potenzen zur neuralen Entwicklung besitzt, eine Erscheinung, die noch spezieller experimenteller Untersuchung bedarf.

\title{
Neue Beobachtungen zum Problem der Flugbahnen bei Hummelmännchen
}

\section{Von Adolf HaAs}

Aus dem Biol. Institut der Philos. Hochschule Berchmanskolleg, Pullach bei München

(Z. Naturforschg. 1, 596-600 [1946]; eingegangen am 24. Juli 1946)

Zu den größten Eigentümlichkeiten und erstaunlichsten Leistungen des Hummellebens gehören eigenartige, streng gesetzmäßige Bahnflüge der Männchen in einem jeweils der betreffenden Hummelart besonders zukommenden Gelände. Über den diesbezüglichen Stand unserer Kenntnisse wird einleitend berichtet. Neue Beobachtungen werden sodann angeführt, welche die Natur dieser Bahnen als Geruchsbahnen erkennen ließen. Anschließend wird auf die Bedeutung des Gesichtssinnes bei diesen Bahnflügen eingegangen und das eigenartige Verhältnis von Geruchsbahn und Gesichtsbahn an Hand verschiedener Beobachtungen diskutiert. Der biologische Sinn der Flugbahnen der Hummelmännchen ist noch vollkommen ungeklärt.

$I^{n}$ m Sommer 1935 entdeckte Arthur Frank ${ }^{1}$ bei Feldkirch (Vorarlberg) eigenartige, bisher unbekannte und auch in der gesamten Biologie der Hymenopteren einzigartige Flugbahnen bei Hummelmännchen. 1941 veröffentlichte er die Ergebnisse $^{2}$.

Die bis dahin beobachteten Tatsachen sind kurz folgende: Sobald die Hummelmännchen ihr Nest für immer verlassen haben, begeben sie sich in einen der jeweiligen Art immer typisch zukommenden Geländeteil (bei Bombus terrestris L. besonders Randgebiete der Wälder). Dort fliegen sie dann eine größere Anzahl ganz bestimmter Geländepunkte der Reihe nach zu einer in sich geschlossenen Bahn ab. Dabei werden die einzelnen Punkte ohne Niedersitzen in einem Abstand von wenigen $\mathrm{cm}$ beflogen. Die Hummel wendet sich dann sofort in meist schnellem, gezieltem Flug dem

1 Gefallen Sept. 1944 in den Beskiden.

nächsten Bahnpunkte zu. Diese Rundflüge auf einer einmal festgelegten Bahn werden über Wochen hinaus beibehalten, ja einzelne Bahnpunkte das ganze Leben hindurch. Die einmal eingeschlagene Flugrichtung wird von jedem einzelnen Individuum bei allen Flügen streng eingehalten. Dabei sind mehrere Bahnpunkte zugleich Anflugspunkte für mehrere Individuen derselben Art, wahrscheinlich auch desselben Nestes. So entsteht an manchen Anflugspunkten oft ein reger Flugverkehr. Die tägliche Flugleistung eines solchen Hummelmännchens (B. terrestris L.) auf der Flugbahn betrug bei günstiger Witterung $60 \mathrm{~km}$. Die Punkte der Flugbahn sind für jede Hummelart typisch verschieden. Es wurde vermutet, daß die einzelnen Punkte Geruchszentren mit zu- und abnehmender Geruchsintensität seien, ohne daß handgreifliche Beweise dafür schon erbracht werden konnten. Ferner blieb ungeklärt, wie-die immer wieder festzustellenden Bahnveränderungen auftreten können.

? Z. vergl. Physiol. 28, 467 [1941]. 
Eingehende Beobachtungen, besonders in den Monaten Juli bis September 1945, förderten nun eine Reihe neuer Tatsachen zutage, über die im folgenden in Form einer vorläufigen Mitteilung kurz berichtet werden soll.

Die Flugbahnen der Hummelmännchen als Geruchsbahnen

Am 3. Juli 1945 machte ich bei Lenting (Ingolstadt) folgende Beobachtung: Nach einem regnerischen Vormittag besserte sich das Wetter. Ich stand an einer Stelle, wo vier Punkte einer Hummelbahn (B.hortorum L.) überblickbar waren. Der erste Punkt befand sich an einem Blatt eines herabhängenden Apfelbaumastes. Die übrigen Punkte lagen entlang einer Efeuhecke. Gegen $11^{\mathrm{h}}$ flog das erste Hummelmännchen. Sein Verhalten war außergewöhnlich. Es kam im langsamen Schwirrfluge an, setzte sich auf das Apfelbaumblatt und umlief eilig den Blattrand, gleichsam auf demselben reitend (Abb.1). Fühler und Kopf des Hummelmännchens waren nach unten geneigt und tasteten dem Blattrand entlang. Danach flog das Männchen zum nächsten Bahnpunkt weiter und wiederholte dort dasselbe eigenartige Spiel an mehreren Efeublättern, die um den Anflugspunkt gruppiert waren. Hierbei wurden alle 5 bis 6 Blätter, die sich um den späteren Anflugspunkt befanden, abgelaufen, und zwar ungefähr in einer Spirale, die von der Mitte des Anflugspunktes sich nach außen erweiterte. Die gleiche Beobachtung war auch beim dritten und vierten Punkt der Bahn zu machen. Nach wenigen Minuten kam das Männchen neuerdings auf seiner Bahn geflogen. Diesmal war der Schwirrflug viel schneller. Es setzte sich nur flüchtig auf das Blatt nieder und glitt, schon halb im Fluge befindlich, die Blattränder entlang. Dasselbe Verhalten auch bei den anderen Bahnpunkten. Nach kurzer Zeit erschien das Hummelmännchen abermals auf der Bahn, und zwar in dem gewöhnlichen, ziemlich schnellen, gezielten Flug, setzte sich nun nicht mehr auf die Anflugstelle nieder, sondern flog dieselbe nur bis auf wenige $\mathrm{cm}$ in der üblichen Weise an und wandte sich dann den übrigen Punkten zu. Von da an war normaler Beflug der Hummelbahn zu beobachten.

Das auffällige Verhalten des Hummelmännchens auf den beiden ersten Flügen legte sofort den Gedanken nahe, daß es sich um ein Kenn- zeichnen der Bahnpunkte mit irgendwelchen Duftstoffen handelte. Schon der Schwirrflug ließ auf eine intensive Tätigkeit von Duftdrüsen schließen ${ }^{3}$.

Am 5. Juli 1945 stand ich bei einer anderen Hummelstraße an einem Bahnpunkt, der sich in der Tiefe eines Farnbusches dicht über dem Boden befand. Kurz vor

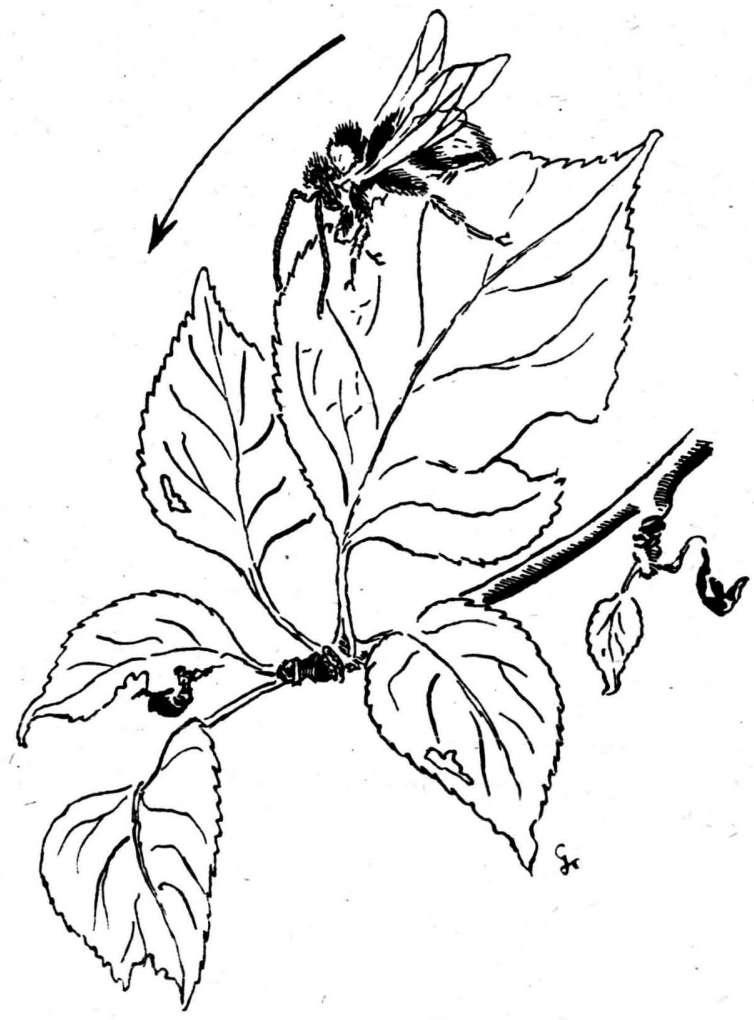

Abb. 1. Hummelmännchen von B. hortorum L., das auf dem Blatt eines Apfelbaumes reitet und dasselbe in der angegebenen Pfeilrichtung mit Duftspuren belegt. (Nach Angaben des Verfassers nach der Natur gezeichnet von Goders ki.)

$9^{\text {h }}$ flog das erste Männchen auf der Bahn. Es flog zuerst in die Tiefe des Farnbusches, setzte sich dort nieder und lief eilig in wenigen, sich nach außen erweiternden Spiralen um den Punkt, flog dann auf den darüber hängenden Himbeerzweig, lief die einzelnen Blattränder ab, um dann das gleiche bei einem noch höher hängenden $\mathrm{Z}$ weige zu tun. Sodann umlief es noch einige Farnblättchen in gleicher Höhe und begab sich dann in dem bekannten schwerfälligen Schwirrfluge zum nächsten Bahnpunkt in der Wurzelnische einer Fichte.

3 Auch bei früheren Beobachtungen hatten wir gelegentlich Hummelmännchen gesehen, die in der angegebenen Weise an Blättern herumkrabbelten (wobei diese Stellen dann unangenehm rochen), ohne daß wir diese Tatsache in Beziehung zu den Hummelbahnen hringen konnten. Vergl. Frank ${ }^{2}$, S. 484. 
Danach kam das Männchen von neuem, zeichnete dieselben Stellen nur flüchtig. Beim dritten Flug endlich war normales Verhalten zu beobachten.

Es war bei dieser und allen späteren Beobachtungen auffällig, daß das Hummelmännchen nur jene Blattränder ablief, die in der Einflugsrichtung lagen. Ferner war das Herumkreisen am untersten Punkte (dem späteren Anflugspunkt) am intensivsten, während mit zunehmendem Abstand vom Anflugspunkt das Herumlaufen auf den Blatträndern flüchtiger wurde. So entstand also augenscheinlich ein Duftzentrum mit einer darüber anschließenden Duftspirale oder einem Duftbogen in der Richtung des Einfluges. Damit erklärt sich die eigenartige, bisher rätselhafte Tatsache, dafs die Hummelmännchen ihre Bahn immer im gleichen Sinne durchfliegen ${ }^{4}$. Aus allen Beobachtungen wurde zugleich ersichtlich, daß das Hummelmännchen zur Bezeichnung der Duftspirale auch Blättchen abläuft. die später nicht unmittelbare Anflugspunkte sind, sondern zu den äußeren Windungen der Duftspirale gehören.

„Spuren“ zeichnende Hummeln (im folgenden immer „Spurhummeln“ genannt) waren immer zu sehen, besonders in den Morgenstunden nach regnerischem Wetter. Im Herbst 1945 wurden die Bahnen von $B$. terrestris L. fast täglich zur selben Zeit von der Spurhummel beflogen.

Daß es sich bei den Spurhummeln, die auf den eigenartigen Hummelbahnen beobachtet werden. um Tiere handelt, die Duftstoffe gleichsam absprühen, wurde durch Abfangen solcher Spurhummeln bewiesen. So fing ich am 21. Juli 1945 eine Spurhummel ( $B$. hortorum L.) im Forstenrieder Park von ihrer Bahn ab. Sie hatte sich an der Wurzelnische einer Fichte niedergelassen und lief dort in Spiralen bis etwa $20 \mathrm{~cm}$ über dem Anflugspunkt. Eine dabeistehende Mycelis-muralisStaude wurde auf der Seite, die sie dem Einflug zuwandte, ebenfalls mitgezeichnet und so in die Duftspirale einbezogen, obgleich sie also später nicht direkt Anflugspunkt war. Die abgefangene Spurhummel gab einen sehr starken, angenehmen, rosenartigen Duft von sich. Der gleiche Duft ging von allen Stellen aus, welche die Spurhummel beim Durchlaufen der Duftspirale begangen hatte. Ein Vergleich mit andern Bodenstellen und Blättern, die nicht beflogen wurden, zeigte den auffallenden Duftunterschied deutlich. Weitere Beobachtungsberichte über die Spurhummel an der gleichen Stelle liegen vor vom 21. Aug. 1945, wo die Spurhummel nach zwei Regentagen um $10^{\mathrm{h}} 30$ die Bahn beflog, ferner vom 22. Aug. 1945, wo die Spurhummel gegen $11^{\mathrm{h}}$ erschien. Am 22. August wurde ganztägig durchlaufend beobachtet ${ }^{5}$. Es

\footnotetext{
4 Vergl. Frank ${ }^{2}$, S. 471.
}

wurde festgestellt, daß die Spurhummel in der Regel nur einmal am Tage, und zwar meist bei den ersten Flügen, die Duftbahnen zeichnet.

Das Vorhandensein einer Spurhummel läßt es auch verständlich erscheinen, wieso immer wieder auf den Hummelbahnen Veränderungen auftreten können ${ }^{6}$. Man kann nämlich häufig feststellen, daß die Spurhummel beim Zeichnen der Flugbahn einen Punkt, den sie am Vortage noch beflogen hatte, ausläßt und gleich den nächsten Punkt oder überhaupt einen neuen mit Duftspuren belegt.

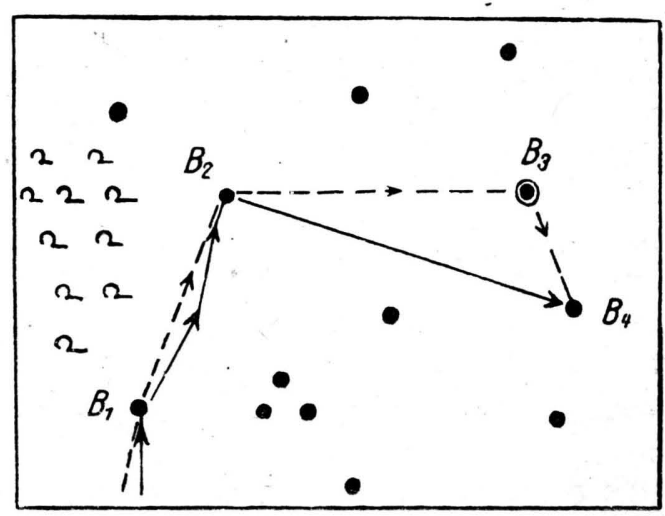

Abb. 2. Teilstrecke aus einer Flugbahn der Hummelmännchen von $B$. terrestris.

$-\rightarrow \rightarrow$ Flugbahn der Hummelmännchen am 22. 8. 45.

$\rightarrow$ Flugbahn am 23.8.45.

Die Punkte stellen Fichten dar.

Als Beispiel diene eine Beobachtung vom 23. Aug. 1945: Am 22. August wurde bei Punkt $B_{3}$ (s. Abb. 2) ganztägig beobachtet. Die Bahn verlief von $B_{1} \rightarrow B_{2}$ $\rightarrow \mathrm{B}_{3} \rightarrow \mathrm{B}_{4} .11^{\mathrm{h}} 01$ erschien ein Hummelmännchen und zeichnete als Spurhummel die Bahn. Es wurde abgefangen. Starker rosenartiger Duft. Es wurde durch ein Stanniołplättchen auf dem Thorax gekennzeichnet. Leider kehrte die gezeichnete Hummel den ganzen Tag nicht wieder. Ein anderes Männchen flog ebenfalls von $11^{\mathrm{h}}$ an die gleiche Bahn. Gegen $17^{\mathrm{h}}$ ging ein heftiges Gewitter nieder, und die ganze Nacht hindurch fielen Gewitterregen. Am nächsten Morgen spärlicher Sonnenschein. Beim Beobachtungsstandort $\mathrm{B}_{3}$ war an diesem Vormittag kein Anflug festzustellen. Punkt $B_{3}$ war verlassen. Dagegen erschien bei $B_{2}$ gegen $11^{\mathrm{h}}$ eine Spurhummel, von $\mathrm{B}_{1}$ kommend, und flog dann direkt nach $B_{4}$, unter Auslassung von $B_{3}$. Diese Bahn $B_{1} \rightarrow$ $\mathrm{B}_{2} \rightarrow \mathrm{B}_{4}$ wurde dann den ganzen Tag beibehalten. Offensichtlich wurde der Punkt $B_{3}$ verlassen, weil er am Morgen nicht mit neuen Duftspuren versehen worden war. Ähnliche Beobachtungen liegen von andern Stellen der Bahn vor.

5 Für selbstlose Hilfe hierbei danke ich Fr. Nitsche. ${ }^{6}$ Vergl. Frank2. S. 478. 
Nach den bisherigen Feststellungen kommen sicher jeder Hummelart und wahrscheinlich auch jeder besonderen Varietät einer Art ganz bestimmte, untereinander verschiedene Duftstoffe zu. Das hauptsächlichste Beobachtungsobjekt sowohl von Frank wie von mir ist eine Varietät von B. terrestris L. und B. hortorum L. mit stark rosenartigem Duft. Eine andere Varietät dieser Hummeln, die ich mehrmals beim „Spuren“ abfing, gab überhaupt keinen deutlich für unser Geruchsvermögen feststellbaren Duft von sich. Am 28. Aug. 1945 beobachtete ich in einem kleinen Wäldchen bei der Garchinger Heide eine Hummelbahn von B. silvarum L. Die abgefangene Spurhummel strömte einen moschusartigen Duft aus. F rank spricht (S.484) von Hummelmännchen, die beim Herumkrabbeln auf den Blättern einen für uns widerlichen Geruch von sich geben. Die Angaben beziehen sich auf gemeinsame Beobachtungen von B.hypnorum. Spurhummeln von $B$. pomorum $\mathrm{Pz}$. fing ich zum erstenmal am 28 . Aug. 1945 auf der Garchinger Heide. Ein auffälliger Geruch war nicht festzustellen.

\section{Gesichts- und Geruchssinn beim Bahnflug}

Wie aus den bisherigen, sich über mehrere Jahre erstreckenden Beobachtungen zur Genüge hervorgeht, sind die Flüge auf den eigenartigen Bahnen als eine der Hauptbeschäftigungen der Hummelmännchen anzusehen. Der Bahnflug wird nur unterbrochen bei gutem Wetter durch meist nur kurze Pausen an sonnigen Plätzchen und auf den Futterpflanzen. Bei ungünstiger Witterung unterbleibt jeder Flug, und die Männchen überstehen diese Zeit an irgendeinem wettersicheren Schlupfwinkel.

Es war von Anfang an wahrscheinlich, daß eine solche, das ganze Leben der Hummelmännchen beherrschende Instinkthandlung nicht nur von einem Sinn allein, dem Geruchssinne, geleitet werden kann. Besonders dem Gesichtssinne mußte eine Bedeutung bei diesen Flügen beigelegt werden. $\mathrm{B}$ i s $\mathrm{ch}$ of $\mathrm{f}^{7}$ weist darauf hin, daß die Entwicklung der Augen bei den Drohnen der Honigbienen, einigen Hummelmännchen (B. confusus Schenck, sicheli $\mathrm{Rad}$.) und andern Hymenopterenmännchen besonders stark ist. $\mathrm{Z}$ an d e r schätzt die Zahl der Facetten bei den weiblichen Honigbienen auf 3-4000, bei den Drohnen jedoch auf 7-8000. $\mathrm{Schmiedeknecht}{ }^{8}$ gibt für Männchen von B. con-

7 Biologie der Hymenopteren, Berlin 1927, S. 170.

8 Die Hymenopteren Mitteleuropas, Jena 1907, S. 40. fusus Schenck „Augen auffallend groß“ an. Eingehende vergleichende Untersuchungen des Auges der Hummelmännchen fehlen uns allerdings noch. Aber schon die angeführten anatomischen Tatsachen deuten auf eine besondere Funktion des Gesichtssinnes hin.

Bei den Beobachtungen der Bahnflüge stellte sich nun folgendes Verhältnis von Geruchs- und Gesichtssinn heraus:

Eine Bahn, die eben von einer Spurhummel beflogen und mit Duftspuren gezeichnet worden war, wurde in den unmittelbar danach erfolgenden Flügen fast nur nach dem Geruchssinne abgeflogen. Das ging daraus hervor, daß sich die bahnfliegende Hummel nur ganz selten durch visuelle Veränderung des Bahnpunktes und dessen näherer Umgebung vertreiben ließ.

Die Spurhummel selbst aber scheint zugleich auch stark vom Gesichtssinne geleitet zu werden. So geschah es oft, daß beim Abfangen der Spurhummel dieselbe entwischte, sei es, daß sie schon das Herannahen des Beobachters bemerkte, sei es, daß die Anwesenheit des Beobachters in nächster Nähe des Anflugpunktes als Störung empfunden wurde. In diesem Falle überging das Hummelmännchen einfach den Anflugspunkt.

Am 18. Juli 1946 wurde ein Bahnpunkt von B. terrestris L. beobachtet. Es war eine Wurzelnische eines Baumstumpfes. Bis $12^{\mathrm{h}} 08$ flogen verschiedene Hummelmännchen jeweils im Abstand von 2-3 Min. an dem Bahnpunkte an. Um die angegebene Zeit legte ich ein weißes Netz über den Stumpf. $12^{\mathrm{h}} 10 \mathrm{kam}$ ein Männchen im gewöhnlichen, ziemlich schnellen Bahnflug an. Jedoch etwa $30 \mathrm{~cm}$ über dem Anflugspunkt stoppte es ruckartig seinen Flug, zog eine Schleife über der Stelle und flog-sichtlich beunruhigt und gestört in Zickzack-Windungen zum nächsten Punkt. Die weiteren Anflüge wurden alle immer flüchtiger. $12^{\mathrm{h}} 18 \mathrm{nahm}$ ich das Netz wieder vom Anflugspunkt weg. Noch in derselben Minute kam ein am Morgen mit „Silber“ gezeichnetes Männchen und flog wieder normal an. Dasselbe auch bei den übrigen Hummelmännchen. Auch aus diesem Experiment geht die Bedeutung des Gesichtssinnes klar hervor.

Durch die zahlreichen Flüge, die ein Hummelmännchen bei günstiger Witterung auf seiner Bahn unternimmt ${ }^{9}$, prägt sich aber immer mehr jeder einzelne Punkt dem außerordentlich gut ausgebildeten visuellen Vermögen der männlichen Hummeln ein. So wird mit der Länge der Flugdauer die Geruchsbahn weitgehend zu einer Ge-

9 Hummelmännchen,,Gold"flog im Juli 1946 die Bahn in der Stunde im Durchschnitt 18-mal ab. Die Flugzeit kann an guten Flugtagen auf mindestens 7 Stdn. berechnet werden. Somit ergibt sich, daß „Gold“ seine Bahn täglich etwa 126-mal durchflogen hat. 
sichtsbahn. Störungen in der Nähe des Anflugspunktes wurden regelmäßig damit beantwortet, daß das Hummelmännchen „Gold“ den visuell ver änderten Anflugspunkt einfach überging und sogleich den nächsten Punkt anflog.

Dennoch handelt es sich bei den Flugbahnen der Hummelmännchen vorwiegend um Geruchsbahnen. Das geht auch daraus hervor, daß Hummelmännchen „Gold“ fast täglich seine Bahn in den Morgenstunden bei den ersten Flügen neu mit Duftstoffen versah. Es ist hier wohl eine kombinierte Tätigkeit der beiden Hauptsinne, des Geruches und des Gesichtes, anzunehmen. Einige Beobachtungen deuten auch darauf hin, daß den Männchen bei ihrem Bahnflug ein ausgebildetes Registriervermögen für bestimmte Strecken, Flugwinkel und bestimmte Zeiten zukommt, worüber in einer späteren Arbeit berichtet werden soll.

\title{
Der Ölgehalt verschiedenchromosomiger Rassen vom Kalmus (Acorus Calamus L.) .
}

\author{
Von Heinz Diedrich WulfF \\ Aus dem Botanischen Institut der Universität Kiel \\ (Z. Naturforschg. 1, 600-603 [1946]; eingegangen am 8. April 1946)
}

\begin{abstract}
Der Gehalt an ätherischem öl wurde bei drei verschiedenen Chromosomenrassen von Acorus Calamus $L$, bestimmt und die folgenden Werte gefunden: Diploide Rasse $(2 n=24) 2,17 \%$, triploide Rasse $(2 n=36) 3,12 \%$, tetraploide Rasse $(2 n=48) 6,82 \%$. Somit nimmt der Gehalt an ätherischem Öl mit steigender Chromosomenzahl zu. Hinsichtlich des Wassergehaltes ergeben diese drei Rassen keine statistisch gesicherte Differenz. Vergleichsweise wurden die Untersuchungen auch auf Acorus gramineus Sol. ausgedehnt. Der Ölgehalt dieser diploiden Art liegt bei $0,7 \%$, ohne daß eine grünblättrige und eine variegate Form Unterschiede zeigten.
\end{abstract}

$I^{n}$ n einer früheren Veröffentlichung ${ }^{1}$ habe ich nachgewiesen, daß beim Kalmus drei Chromosomenrassen vorkommen, nämlich eine diploide mit $2 n=24$, eine tetraploide mit $2 n=48$ und eine triploide mit $2 n=36$ Chromosomen. Diese letztere ist die vor allem in der nördlichen Hemisphäre weitverbreitete sterile Rasse, die nur zu vegetativer Vermehrung befähigt ist, da es infolge der Triploidie nicht zur Bildung funktionsfähiger Samenanlagen und Pollenkörner kommen kann. Ihre Chromosomenzahl $2 n=36$ wurde von $\mathrm{O}$. $\mathrm{Palmg} \mathrm{ren}{ }^{2}$ auch für wildwachsende Kalmuspflanzen Schwedens ermittelt. Die untersuchten diploiden und tetraploiden Pflanzen habe ich dagegen aus Samen herangezogen, die aus den Botanischen Gärten in Kopenhagen bzw. Leningrad stammten. Uber das Wildvorkommen dieser beiden Rassen können zur Zeit keine über bloße Vermutungen hinausgehenden Ansichten geäußert werden. Außer den drei Rassen von Acorus Cala-

1 Planta 31, 478 [1941]

= Bot. Not. 1943. 348 . mus $L$. ist weiterhin der habituell stark abweichende Acorus gramineus Sol. durch die Zählung G. Nakajima s${ }^{3}$, die ich bestätigen konnte, chromosomal als diploid bekannt gemacht worden $(2 n=24)$. Aus der aufgeführten orthoploiden Reihe der Chromosomenzahlen fällt allerdings der in Nordamerika einheimische fertile Kalmus heraus. Nach Untersuchungen von $\mathrm{D}$ a h $\mathrm{l}$, die von M. G. D u d l e $y^{4}$ und M. F. B u e $11^{5}$ zitiert werden, besitzt diese interessante Kalmusrasse nur $2 n=18$ Chromosomen. Es wird notwendig sein, sie späterhin vergleichsweise noch eingehender zu studieren.

Ohne damit eine prinzipielle Stellungnahme ausdrücken zu wollen, hatte ich mich in meiner früheren Arbeit der landläufigen Ansicht angeschlossen, daß die Ahnen der heute in Westeuropa heimisch gewordenen Kalmuspflanzen aus kleinasiatischen Standorten über Konstantinopel-Wien eingeführt worden seien. Für Osteuropa wurde daneben bislang noch

3 Jap. J. Gen. 9, 1 [1933].

4 Bot. Gaz. 98, 556 [1937].

s Bot. Gaz. 99, 556 [1938]. 